\title{
Elternerwartungen an Vorschulkinder: Eine empirische Studie zu ihrer Rolle und ihren Determinanten
}

\section{Margrit Stamm und Doris Edelmann}

Es ist eine empirisch vielfach belegte Tatsache, dass Eltern mit ihrem Verhalten einen grossen Einfluss auf die Entwicklung ihres Kindes haben. Zur Frage jedoch, welche Rolle ihre Erwartungshaltungen spielen, liegen gerade für den Vorschulbereich nur wenige Untersuchungen vor. Dieser Aufsatz präsentiert ausgewählte Daten aus einer Schweizer Pilotstudie zu Erwartungen von Vorschuleltern. In einer Stichprobe von $N=200$ Müttern und $N=200$ Vätern zeigten letztere signifikant höhere Erwartungen als Mütter an die Entwicklung der akademischen, motorisch-sportlichen und künstlerisch-musikalischen Fähigkeiten ihres Kindes, nicht jedoch an die Entwicklung sozialer Verhaltensweisen. Die wichtigste Variable, welche die Elternantworten beeinflusste, war die primäre Betreuungszeit. Eltern, welche mehr als fünfzehn Stunden pro Woche mit ihrem Kind verbrachten, hatten grössere Erwartungen an die Fähigkeits- und Verhaltensentwicklung ihres Kindes als Eltern mit einem geringeren Ausmass primärer Betreuungszeit.

Im Zuge der Etablierung frühkindlicher Förderprogramme gibt es eine wachsende Diskussion zur Frage, welche Bedeutung dem Elternengagement und der mit dem Kind gemeinsam verbrachten Zeit zukommt. Die Meinungen sind dabei geteilt. Eher psychoanalytisch orientierte Fachleute befürchten, dass frühe Förderung, insbesondere wenn sie kognitiv orientiert und familienextern erfolgt, einer gesunden kindlichen Entwicklung eher abträglich ist (Dornes, 2009; Largo, 2009). Die Hirnforschung wiederum unterstreicht die enorme Bedeutung des gezielten, frühen elterlichen Förderengagements, weil es die hirnbiologische Basis für spätere Lernleistungen und sozio-emotionale Kompetenz bildet (Spitzer, 2010). Solche Meinungsverschiedenheiten haben der Frage neuen Aufschwung verliehen, inwiefern Eltern ihr Vorschulkind schon früh zum Erwerb von Wissen und Können drängen können, das normalerweise erst auf einem späteren Entwicklungsniveau erworben wird. Elkind hat diese Problematik bereits in den 1980er-Jahren unter Stichworten wie "The hurried child» (deutsch: "Das gehetzte Kind», 1988) oder «Miseducation» (deutsch: "Wenn Eltern zuviel fordern», 1989) diskutiert und dabei auch auf 
die mit Elterndruck und übertriebenen Erwartungshaltungen verbundenen Entwicklungsrisiken verwiesen. Die damals auf die USA beschränkte Aufmerksamkeit (Gallagher \& Coche, 1987; Hills, 1987; Sigel, 1987) hat nun neuerdings auch hierzulande unter dem Stichwort «Treibhaus-Debatte» Eingang in die wissenschaftliche und gesellschaftspolitische Diskussion gefunden (Largo, 2009; Stamm, 2010; Schmitt, Kuger, Kluczniok \& von Maurice, 2010). Dieses «Treibhaus-Phänomen» ist empirisch bisher allerdings kaum untersucht.

Hier setzt der vorliegende Beitrag ein. Basierend auf der Annahme, dass Eltern mit hohen Erwartungshaltungen auch entwicklungsförderliche Lernumgebungen schaffen (Klein \& Biedinger, 2009; Schmitt, 2009; Ebbeck, 2010), untersucht er ihre normativen Vorstellungen, was ihr Kind im Hinblick auf den Schuleintritt können und lernen soll. Im Mittelpunkt steht dabei die Frage, wie ausgeprägt und wie spezifisch die Erwartungshaltungen sind und inwiefern sich zwischen Müttern und Vätern Unterschiede abzeichnen (Fragestellung 1) sowie welche Effekte Geschlecht und Alter des Kindes sowie Ausbildung, Haushaltseinkommen der Eltern und die primäre Betreuungszeit spielen (Fragestellung 2).

\section{Theoretische und empirische Grundlagen}

In der internationalen Forschung zur Entwicklung von Vorschulkindern gelten das familiäre Umfeld und die gemeinsam verbrachte Zeit von Eltern und Kindern schon seit längerem als massgebliche Ursachen für eine gesunde Entwicklung der Kinder. Folgt man Coleman (1988), so sind die Erwartungshaltungen von Eltern eine wichtige Komponente des Humankapitals, welches zusammen mit den ökonomischen Ressourcen und dem Sozialkapital den Familienhintergrund eines Kindes abbildet. Das Humankapital steht dabei für eine kognitiv anregende Atmosphäre, den zeitlichen Umfang, den Eltern ihrem Kind zuteil kommen lassen, wie auch für die elterlichen Erwartungshaltungen. Hierzu existieren im englischsprachigen Raum einige interessante Untersuchungen (vgl. zusammenfassend Hyson, Hirsh-Pasek, Rescorla, Cone \& Martell-Boinske, 1990). Sigel und McGilluddy-DeLisi (1982) wiesen beispielsweise nach, dass Eltern, die ihr Kind gezielt auf die Schule vorbereiten, seine Initiative und Kreativität, aber auch seine Fähigkeit zum Problemlösen hemmen. Hyson et al. (1990) oder Rescorla, Hyson und Hirsh-Pasek (1991) untersuchten anhand der Educational Attitude Scale (EAS) das Ausmass der Elternerwartungen in verschiedenen Fähigkeitsbereichen und im sozialen Verhalten. Dabei zeigten sich grosse Unterschiede in den schulvorbereitenden Erwartungshaltungen, nicht jedoch im Hinblick auf das soziale Verhalten.

In der deutschsprachigen Forschung liegen zu Elternerwartungen bislang nur wenig empirische Untersuchungen vor, obwohl der «Bildungsort Familie» zu einem äusserst vielfältig verwendeten Begriff geworden ist (z. B. Büchner, 2011). Ein Grossteil der vorliegenden Studien beschäftigt sich hauptsächlich damit, wie 
Erwartungshaltungen im Sinne von Bildungsaspirationen überhaupt zustande kommen und welche Einflüsse damit verbunden sind. Dabei zeigt sich, dass die Bildung der Eltern sowie ihre ökonomische Situation (Schilther, 2012) die Erwartungshaltungen stark beeinflussen (Ditton, Krüsken \& Schauenberg, 2005; Kurz \& Paulus, 2006; Paulus \& Blossfeld, 2007; Schuchart \& Maaz, 2007). Zur auf Bourdieu (1982) rekurrierenden Annahme, dass sich das Interesse am Statuserhalt auf die Erwartungshaltungen von Familien auswirkt und dabei auch materielle Güter und Bildungstitel eine Rolle spielen, liegen vielfache empirische Erkenntnisse vor (Jungbauer-Gans, 2004; Rössel \& Beckert-Zieglschmid, 2002; van de Werfhorst \& Hofstede, 2007). Von Lareau (2003) wissen wir ferner, dass gerade bildungsnahe Familien die ersten Lebensjahre der Kinder nicht nur sorgfältig im Sinne einer concerted cultivation ausgestalten, sondern auch mit hohen Erwartungen versehen. Derartige Einstellungen verweisen gemäss Kurz und Paulus (2006) auf das Interesse am Statuserhalt, der sich wiederum positiv auf die Erwartungshaltungen auswirkt.

Gemäss Bourdieu (1982) ist für die Akkumulation von kulturellem Kapital eine Befreiung von finanziellen Zwängen erforderlich, weshalb ein gewisses Mass an ökonomischem Kapital erforderlich ist. Als Indikatoren hierfür gelten das Durchschnittseinkommen oder das Wohnungseigentum. Die Befunde hierzu sind kontrovers. In einigen Studien (beispielsweise Zick, Bryant \& Österbacka, 2001) zeigt sich ein positiver, in anderen Studien kein direkter (Walter \& Künzler, 2002) oder ein negativer Zusammenhang (Craig, 2006).

Folgt man Coleman (1988), so spielt neben hohen elterlichen Erwartungshaltungen auch die gemeinsam verbrachte Zeit (Betreuungszeit) für ein gesundes Aufwachsen eine wichtige Rolle. Sie wird in primäre und sekundäre Betreuungszeit aufgeteilt (Zick \& Bryant, 1996; Walter \& Künzler, 2002). Erstere umfasst die aktive Kinderbetreuung, letztere wird als Zeit verstanden, bei der die Hauptaufmerksamkeit der Eltern auf einer anderen Tätigkeit liegt und das Kind nur passiv betreut wird. Gemäss Craig (2006) oder Walter und Künzler (2002) spielt dabei die Ausbildung der Eltern insofern eine Rolle, als sich ein positiver Zusammenhang mit der gemeinsam verbrachten Zeit ergibt. So verbringen Eltern mit akademischem Ausbildungshintergrund pro Tag eine Viertelstunde mehr mit ihrem Kind als Nicht-Akademiker-Eltern und gestalten diese auch deutlich entwicklungsförderlicher (Bianchi, 2000). Inwiefern und ob höhere finanzielle Ressourcen die primäre Betreuungszeit (die ja auch kostenintensiv sein kann) überhaupt erst ermöglichen oder dazu beitragen können, bleibt insgesamt unklar. Folgt man Petzold und Nickel (1989), Dornes (2009), Lamb (2004), der NICHD-Studie (2008) oder den umfassenden Studien von Grossmann und Grossmann (2012) sowie den Untersuchungen zum Erziehungsverhalten von Müttern und Vätern von Franiek und Reichle (2007) respektive zum Väterhandeln von Grunow (2007), so kann davon ausgegangen werden, dass Väter sowohl das Motorische stärker gewichten als auch die Autonomie und deshalb in diesen Bereichen andere Erwartungen haben dürften als die Mütter. 
Schliesslich stellt sich die Frage, inwiefern das Alter des Kindes eine Rolle spielt. Zwar liegen einige wenige Erkenntnisse vor, die darauf verweisen, dass Eltern unmittelbar vor dem Schuleintritt besonders hohe Erwartungen aufbauen, doch stammen sie entweder aus älteren amerikanischen Studien (Hyson et al., 1990; Sigel \& McGilluddy-DeLisi, 1982) oder dann beziehen sie sich auf Bildungsaspirationen im Sinne von Erwartungen, ob das Kind das Abitur erreicht. In dieser Untersuchung hat sich diese Operationalisierung insgesamt als schwierig erwiesen, da sich viele Eltern hierzu noch gar keine Gedanken gemacht hatten.

Zusammengefasst verweist die Forschung auf die enorme Bedeutung der Elternerwartungen, die im Kontext des Ausbildungsniveaus, der primären Betreuungszeit sowie des Haushaltseinkommens diskutiert werden müssen. Allerdings ist nahezu unbekannt, ob das Alter des Kindes eine Rolle spielt, d. h. ob Eltern von Kindern, die vor der Einschulung stehen, andere oder höhere Erwartungshaltungen haben als Eltern jüngerer Kinder. Zweitens wurden die Einstellungen der Väter bisher kaum erhoben. Aus der verfügbaren Forschung wissen wir jedoch, dass Väter anders mit ihren Kindern interagieren und dies auch anders gewichten. Insgesamt wird erwartet, dass Eltern von Vorschulkindern besonders hohe Erwartungshaltungen haben und dass sich diesbezüglich Väter von Müttern unterscheiden.

\section{Untersuchungsdesign}

\section{Stichprobe und Ablauf}

Grundlage der hier präsentierten explorativen Studie bildet ein im Jahr 2008 erhobener Datensatz zu den Erwartungshaltungen von Vorschuleltern. Grundlage bildete die Austestung der deutschen Fassung der Educational Attainment Scale (EAS) von Rescorla et al. (1991). Über die Zusammenarbeit mit 35 Kindergärten aus drei Deutschschweizer Kantonen konnten N = 298 Familien zur Beteiligung eingeladen werden. $\mathrm{N}=222$ Familien sagten schliesslich $\mathrm{zu}$. Ihnen wurde durch ihr Kind, das zum Zeitpunkt der Untersuchung zwischen viereinhalb und sechs Jahre alt war, der EAS-Fragebogen sowie der Fragebogen zu demografischen Variablen nach Hause gebracht. Dabei wurden die Mütter und Väter gebeten, je individuell den Fragebogen auszufüllen und sich dabei gegenseitig nicht zu konsultieren. Da schliesslich $\mathrm{N}=222$ Mütter, jedoch nur $\mathrm{N}=200$ Väter den EAS-Fragebogen tatsächlich ausfüllten, wurden letztlich nur die Datensätze der vollständigen Paare (d. h. $N=200$ Mütter und $N=200$ Väter, $\sum: N=400$ ) und ihrer Kinder ( $\mathrm{N}=103$ Jungen; $\mathrm{N}=97$ Mädchen) in die Auswertung einbezogen.

\section{Instrumente}

Elterliche Erwartungshaltungen: Zur Erfassung von Elternerwartungen wird am häufigsten auf die Operationalisierung in Form des Wunsches der Eltern zurückgegriffen, inwiefern sie möchten, dass ihr Kind das Abitur / die Maturität 
erreicht. Da es jedoch schwierig ist, für Vorschulkinder eine solche Messung zu erfassen, weil sich viele Eltern zu diesem Zeitpunkt noch gar keine Gedanken dazu gemacht haben, wurde als Alternative die deutsche Fassung der Educational Attainment Scale EAS von Rescorla et al. (1991) eingesetzt. Dabei handelt es sich um ein Likert-skaliertes Instrument mit 28 positiv und negativ gepolten Aussagen in Bezug auf Elternerwartungen in drei Bereichen (schulvorbereitend [Beispielitem: «Ich erwarte, dass mein Kind zu zählen lernt, auch wenn es sich nicht richtig interessiert»]; motorisch/sportlich [Beispielitem: «Unser Kind ist zu jung, um schwimmen zu lernen»]; künstlerisch/musikalisch [Beispielitem: «Ich erwarte von unserem Kind, dass es sich dafür interessiert, ein Instrument spielen zu lernen»]) und in zwei sozialen Bereichen (soziales Verhalten [Beispielitem: «Mein Kind soll lernen, auch mit anderen auszukommen»], Anpassungsfähigkeit [Beispielitem: «Ich erwarte, dass unser Kind die Tischmanieren übernimmt»]). Jede der Aussagen schliesst sechs mögliche Antworten ein, die von überhaupt nicht einverstanden $(=1.0)$ bis sehr einverstanden $(=6.0)$ reichen. Ein hoher Wert bedeutet somit, dass Eltern schulvorbereitende Fähigkeiten, erwachsenengesteuerte Anleitungen und die Bedeutung des Erwerbs motorisch/sportlicher und künstlerisch/musikalischer Fähigkeiten betonen.

Ausbildung der Eltern: Die Ausbildung der Eltern wurde mittels sechs Kategorien (obligatorische Schule, Anlehre, Berufslehre, Berufslehre inkl. Meisterdiplom, Höhere Fachschule, Fachhochschule/Universität/ETH) erfasst. Aufgrund der minimalen Besetzung einzelner Kategorien wurden sie schliesslich zu vier Kategorien zusammengefasst (vgl. Tabelle 1). Gemäss dem Bundesamt für Statistik (2009b) gehören zur untersten Kategorie $13 \%$, zur Kategorie Berufslehre inkl. Meisterdiplom 50 \%, zur Kategorie Höhere Berufsbildung $17 \%$ und zur Kategorie Hochschule/Universität $20 \%$.

Haushaltseinkommen: Das Einkommen dient als Indikator der (materiellen) Wohlfahrt. Deshalb wurden die Befragten gebeten, das Jahreseinkommen insgesamt anzugeben und sich einer von drei Einkommenskategorien (verfügbares Einkommen) zuzuordnen: weniger als $4000 \mathrm{CHF}$, zwischen $4000 \mathrm{CHF}$ und 7000 CHF und mehr als 7000 CHF monatlich. Da neben der Anzahl der Haushaltsmitglieder auch das Alter einen Einfluss auf den effektiven Bedarf hat, wurde das Äquivalenzeinkommen gemäss den Skalen des Bundesamtes für Statistik (2009a) berechnet. Dieses wird ermittelt, indem die einzelnen Haushaltsmitglieder gewichtet werden: Die erste erwachsene Person mit 1.0, die zweite und jede weitere im Alter von 14 und mehr Jahren mit 0.5 sowie jedes Kind unter 14 Jahren mit 0.3 (entspricht der modifizierten OECD-Äquivalenzskala). Gemäss diesen Angaben betrug es im Jahr 20094152 CHF. $20.4 \%$ der Haushalte gehörten zur ersten, $50.2 \%$ zur zweiten und $25.1 \%$ zur dritten Kategorie.

Primäre Betreuungszeit: Die primäre Betreuungszeit (als der Zeit, in der die Betreuung und Förderung des Kindes die Hauptaufgabe bildet) wurde bei der Hauptbetreuungsperson (die zu 94.5\% die Mutter war) mit drei Ausprägungen erfasst (weniger als 10 Std. pro Woche, zwischen 10 und 15 Std. pro 
Woche, mehr als 15 Std. pro Woche). Die primäre Betreuungszeit beträgt in Deutschland durchschnittlich 15 Stunden pro Woche für Mütter, 9.5 Stunden für Väter (Hofferth, 2001; Künzler, Walter, Reichart \& Pfister, 2001).

\section{Datenanalysen}

In einem ersten Schritt wurden verschiedene deskriptive Analysen durchgeführt, um die Item-Häufigkeiten, Prozent- und Mittelwerte sowie Standardabweichungen zu berechnen. Die unabhängigen Variablen schlossen das Geschlecht der Eltern, das Geschlecht und das Alter des Kindes, die primäre Betreuungszeit, die Ausbildung der Eltern und das Haushaltseinkommen ein. Die abhängigen Variablen umfassten die fünf Subskalen der EAS. In einem zweiten Schritt wurden Varianzanalysen für jede Subskala durchgeführt, um die Effekte jeder unabhängigen Variablen auf jede Subskala zu bestimmen. Paarweise Vergleiche der Items sollten ferner signifikante Unterschiede in den Subskalen eruieren. Multivariate Varianzanalysen wurden durchgeführt, um die Effekte der drei Niveaus der primären Betreuungszeit, der drei Alterskategorien des Kindes, der vier elterlichen Ausbildungsniveaus sowie der drei Kategorien des Haushaltseinkommens auf die fünf abhängigen Variablen zu berechnen.

\section{Ergebnisse}

Der Tabelle 1 sind die Häufigkeitsauszählungen zu entnehmen. Dabei zeigt sich, dass Jungen und Mädchen in der Stichprobe ausgewogen vertreten sind. Gleiches gilt für das Alter der Kinder: 31 \% Vierjährige stehen $34 \%$ Fünf- und 35 \% Sechsjährigen gegenüber. In Bezug auf die primäre Betreuungszeit berichteten $40 \%$, dass sie das Kind mehr als 15 Stunden pro Woche primär betreuen, weitere $37 \%$ zwischen 10 und 15 Stunden und $24 \%$ weniger als 10 Stunden. Damit liegen diese Werte leicht höher als sie für Deutschland festgehalten sind (Hofferth, 2001; Künzler et al., 2001).

Im Hinblick auf das Ausbildungsniveau der Befragten verfügten $13 \%$ über einen Abschluss der obligatorischen Schule oder eine Anlehre, $47 \%$ über eine Berufslehre oder ein Meisterdiplom, 19 \% über eine höhere Berufsausbildung und $21 \%$ über einen Hochschulabschluss. Im Vergleich zur statistischen Verteilung in der Schweiz, die einen Anteil von 24.6\% akademisch Gebildeter ausweist (Bundesamt für Statistik, 2009b), ist er in unserer Stichprobe mit 31.3 \% deutlich höher. Da auch das Haushaltseinkommen etwas höher ausfiel als dies für die Schweiz im Jahr 2009 durchschnittlich der Fall war (Bundesamt für Statistik, 2009a) - die beiden oberen Kategorien waren mit 52 \% (Einkommen zwischen $4000 \mathrm{CHF}$ und $7000 \mathrm{CHF}$ ) sowie $29 \%$ (über 7000 $\mathrm{CHF}$ überrepräsentiert - lässt sich insgesamt von einer tendenziell «bildungsnahen» Stichprobe sprechen. 
Tabelle 1: Häufigkeiten und Prozentwerte der demografischen Variablen

\begin{tabular}{lrc}
\hline & Häufigkeit & Prozent \\
\hline Geschlecht des Kindes $(N=200)$ & 103 & 50.3 \\
Jungen & 97 & 49.7 \\
Mädchen & & \\
\hline Alter des Kindes $(N=200)$ & 61 & 30.5 \\
$\quad$ vier Jahre & 69 & 34.5 \\
fünf Jahre & 70 & 35.0 \\
sechs Jahre & & \\
\hline Primäre Betreuungszeit $(N=200)$ & 48 & 23.9 \\
10 Stunden oder weniger & 73 & 36.5 \\
11-15 Stunden & 79 & 39.6 \\
$\quad$ mehr als 15 Stunden & & \\
\hline Ausbildung der Eltern $(N=400)$ & 58 & 14.5 \\
obligatorische Schule/Anlehre & 135 & 33.7 \\
Berufslehre inkl. Meisterdiplom & 82 & 20.5 \\
Höhere Berufsbildung & 125 & 31.3 \\
Fachhochschule/Universität/ETH & & \\
\hline Haushaltseinkommen (Äquivalenzeinkommen) $(N=400)$ & 55 & 13.8 \\
4000 CHF oder weniger & 226 & 56.5 \\
4000 CHF - 7000 CHF & 119 & 29.7 \\
mehr als 7000 CHF & &
\end{tabular}

Fragestellung 1: Wie ausgeprägt sind die elterlichen Erwartungen und zeigen sich zwischen Müttern und Vätern Unterschiede?

Die erste Analyse ist den mittels der EAS erhobenen elterlichen Erziehungserwartungen gewidmet. Aus Tabelle 2 wird aus der Spalte «kombiniert» ersichtlich, dass die Elternerwartungen im sozialen Verhalten $(M W=5.20)$ und in der Anpassungsfähigkeit $(M W=4.78)$ höher und die Bandbreite kleiner $(S D$ $=0.43$ resp. 0.55$)$ als in den drei anderen Bereichen Schulvorbereitung $(M W=$ $4.42, S D=1.02)$, Motorik/Sport $(M W=4.06 ; S D=0.70)$, Kunst/Musik $(M W$ $=3.34 ; S D=0.64)$ war. In den schulbezogenen Erwartungen waren die Unterschiede mit $S D=1.15$ besonders gross. Vergleicht man die Werte von Müttern und Vätern miteinander, so erweisen sich letztere als insgesamt anspruchsvoller $(t(400)=-4.01, p<.001)$. Die befragten Väter erwarteten vor allem in der Schulvorbereitung $(M W=4.40)$, in Motorik/Sport $(M W=4.32)$ sowie in Kunst/Musik $(M W=3.59)$ deutlich mehr von ihren Kindern als die Mütter $(M W=3.39 ; 3.66 ; 3.10)$. Im sozialen Verhalten und der Anpassungsfähigkeit unterschieden sich Väter $(M W=5.22 ; 4.73)$ von den Müttern $(M W=5.29$; 4.73) jedoch nur zufällig. 
Tabelle 2: EAS-Mittelwerte (MW) und Standardabweichungen (SD) nach Bereich für Väter und Mütter

\begin{tabular}{lcccccccc}
\hline \multirow{2}{*}{ Bereich } & \multicolumn{2}{c}{ Mütter } & \multicolumn{2}{c}{ Väter } & \multirow{2}{c}{ kombiniert } \\
\cline { 2 - 4 } & $M W$ & $S D$ & $M W$ & $S D$ & & & $M W$ & $S D$ \\
\hline Schulvorbereitung & 3.39 & 1.15 & 4.40 & 1.14 & $-5.62^{* *}$ & 4.42 & 1.12 \\
Motorik/Sport & 3.66 & 0.91 & 4.32 & 0.91 & $-3.32^{* *}$ & 4.06 & 0.70 \\
Kunst/Musik & 3.10 & 0.86 & 3.59 & 0.82 & $-3.49^{* *}$ & 3.34 & 0.64 \\
Soziales Verhalten & 5.29 & 0.44 & 5.22 & 0.59 & 1.10 & & 5.20 & 0.43 \\
Anpassungsfähigkeit & 4.73 & 0.59 & 4.73 & 0.71 & -0.05 & & 4.78 & 0.55 \\
\hline Total $^{\circ}$ & 4.09 & 0.52 & 4.43 & 0.62 & $-4.01^{* * *}$ & 4.25 & 0.45 \\
\hline
\end{tabular}

$N=200$ Mütter; $N=200$ Väter; ${ }^{*} p<.05 ;{ }^{* *} p<.01 ;{ }^{* * *} p<.001$; empirischer Wertebereich $=1.00$ bis 6.00 für Schulvorbereitung, Motorik/Sport sowie Kunst/Musik; empirischer Wertebereich $=3.00$ bis 6.00 für Sozialkompetenz und Anpassungsfähigkeit; ${ }^{\circ}$ additive Skala, gemittelter Wert

\section{Fragestellung 2: Welche Rolle spielen Geschlecht und Alter des Kindes sowie Ausbildung, Haushaltseinkommen und die primäre Betreuungszeit?}

In einem nächsten Schritt wurden für jede abhängige Variable zur Bestimmung von Effekten der verbleibenden unabhängigen Variablen (Geschlecht und Alter des Kindes, primäre Betreuungszeit, Ausbildung der Eltern, Haushaltseinkommen) weitere Varianzanalysen durchgeführt. Wie aus Tabelle 3 ersichtlich wird, konnten für die unabhängigen Variablen Geschlecht des Kindes und Haushaltseinkommen in allen EAS-Subskalen keine signifikanten Unterschiede gefunden werden, wohl jedoch für die Variablen des Alters, der primären Betreuungszeit sowie der Ausbildung der Eltern. Aus diesem Grund wurden multivariate Varianzanalysen gerechnet, um die Effekte der unterschiedlichen Ausprägungsgrade dieser Variablen auf die abhängigen Variablen zu eruieren (Spalte 2 in Tabelle 3).

Tabelle 3: Ergebnisse der Varianzanalysen zu den abhängigen und unabhängigen Variablen

\begin{tabular}{lcclllll}
\hline & & \multicolumn{5}{c}{ univariate $F$} \\
\cline { 3 - 7 } Unabhängige Variablen & $\begin{array}{c}\text { Multi- } \\
\text { variate } F\end{array}$ & $\begin{array}{l}\text { Schulvor- } \\
\text { bereitung }\end{array}$ & $\begin{array}{l}\text { Motorik/ } \\
\text { Sport }\end{array}$ & $\begin{array}{l}\text { Kunst/ } \\
\text { Musik }\end{array}$ & $\begin{array}{l}\text { Soziales } \\
\text { Verhalten }\end{array}$ & $\begin{array}{l}\text { Anpas- } \\
\text { sungs- } \\
\text { fähigkeit }\end{array}$ \\
\hline Geschlecht des Kindes & 0.48 & 0.10 & 1.25 & 1.58 & 0.15 & 1.23 \\
Alter des Kindes & 0.88 & $8.25^{* *}$ & 6.66 & 0.16 & 4.55 & 3.41 \\
Primäre Betreuungszeit & $25.14^{* * *}$ & $15.95^{* * *}$ & $4.50^{*}$ & $7.70^{* *}$ & $13.25^{* *}$ & $13.03^{* *}$ \\
Haushaltseinkommen & 0.19 & 2.55 & 0.38 & 2.12 & 1.99 & 1.34 \\
Ausbildung der Eltern & $7.54^{*}$ & $13.25^{* *}$ & 1.66 & $3.05^{*}$ & 2.44 & $5.72^{* *}$ \\
\hline
\end{tabular}

$N=200$ Mütter; $N=200$ Väter; ${ }^{*} p<.05 ;{ }^{* *} p<.01 ;{ }^{* * *} p<.001$; empirischer Wertebereich $=1.00$ bis 6.00 für Schulvorbereitung, Motorik/Sport sowie Kunst/Musik; empirischer Wertebereich $=3.00$ bis 6.00 für Sozialkompetenz und Anpassungsfähigkeit 


\section{Geschlecht und Alter des Kindes}

Das Geschlecht erwies sich gemäss der multivariaten Varianzanalyse als unbedeutsame Variable $(F(12,600)=0.48 ; p=.122)$. In der Tendenz gilt dies auch für das Alter des Kindes, doch weist die für jede Subskala und den Gesamtwert durchgeführte Analyse einen signifikanten Einfluss der Elternerwartungen in Bezug auf die Schulvorbereitung $(F(4,400)=8.25, p<.01)$ nach. Dabei verwiesen die anschliessend anhand des Tukey-Tests, der aufgrund des signifikanten Globaleffekts der Varianzanalyse in Tabelle 3 gewählt wurde, durchgeführten paarweisen Vergleiche der Mittelwertsunterschiede in den drei Alterskategorien auf signifikant anspruchsvollere Erwartungshaltungen der Eltern sechsjähriger Kinder $(M W=3.91, S D=0.59 ; p<.05)$. Sie unterschieden sich deutlich von den Erwartungshaltungen der Eltern vierjähriger Kinder $(M W=3.70 ; S D=0.67 ; p<.01)$.

\section{Primäre Betreuungszeit}

Auf der Basis des Wilks-Kriteriums erwiesen sich die kombinierten abhängigen Variablen als signifikant beeinflusst von der primären Betreuungszeit $(F(8,400)$ $=27.14, p<.05)$. Auch die nachfolgenden Varianzanalysen - als Follow-Up der multivariaten Varianzanalyse durchgeführt - förderten für drei der Subskalen signifikante Ergebnisse zutage (vgl. Tabelle 4): für die Schulvorbereitung $(F(8$, $400)=15.95, p<.01)$, Motorik/Sport $(F(8,400)=35.68, p<.01)$ sowie Kunst $/$ Musik $(F(8,400)=26.14, p<.05)$.

Tabelle 4: EAS-Mittelwerte (MW), Standardabweichungen (SD) und Werte der Varianzanalysen für die drei Ausprägungen der primären Betreuungszeit

\begin{tabular}{|c|c|c|c|c|c|c|c|c|}
\hline \multirow{3}{*}{ Bereich } & \multicolumn{8}{|c|}{ Primäre Betreuungszeit } \\
\hline & \multicolumn{2}{|c|}{$\begin{array}{c}\text { weniger als } \\
10 \mathrm{Std} \text {. }\end{array}$} & \multicolumn{2}{|c|}{11 bis 15 Std. } & \multicolumn{2}{|c|}{ mehr als 15 Std. } & \multirow{2}{*}{$\begin{array}{l}F(8, \\
600)\end{array}$} & \multirow{2}{*}{$\mathrm{p}$} \\
\hline & $M W$ & $S D$ & $M W$ & $S D$ & $M W$ & $S D$ & & \\
\hline Schulvorbereitung & 3.82 & 0.88 & 4.04 & 0.55 & 4.21 & 0.45 & 15.95 & $0.009^{* *}$ \\
\hline Motorik/Sport & 3.54 & 0.54 & 3.99 & 0.57 & 4.32 & 0.55 & 35.68 & $0.002^{* *}$ \\
\hline Kunst/Musik & 3.34 & 0.64 & 3.47 & 0.86 & 3.74 & 0.56 & 26.14 & $0.013^{* *}$ \\
\hline Soziales Verhalten & 5.19 & 0.44 & 5.20 & 0.33 & 5.27 & 0.40 & 4.55 & 0.111 \\
\hline Anpassungsfähigkeit & 4.76 & 0.56 & 4.89 & 0.70 & 4.80 & 0.44 & 8.27 & 0.122 \\
\hline Total $^{\circ}$ & 4.03 & 0.55 & 4.10 & 0.63 & 4.12 & 0.50 & 27.14 & $0.019^{*}$ \\
\hline
\end{tabular}

$N=200$ Mütter; $N=200$ Väter; ${ }^{*} p<.05 ;{ }^{* *} p<.01 ;{ }^{* * *} p<.001$; empirischer Wertebereich $=1.00$ bis 6.00 für Schulvorbereitung, Motorik/Sport sowie Kunst/Musik; empirischer Wertebereich $=3.00$ bis 6.00 für Sozialkompetenz und Anpassungsfähigkeit; ${ }^{\circ}$ additive Skala, gemittelter Wert

Die Varianzanalyse zu den Effekten der drei Niveaus der primären Betreuungszeit verdeutlicht ihren Einfluss auf den Gesamtwert $($ Total $)(F(8,400)=$ 27.14, $p<.05)$. Um des Weiteren die Beziehung zwischen den Gruppen zu verstehen, wurden wiederum paarweise Vergleiche der Mittelwertsunterschiede 
für die Zeitvariable durchgeführt. Eltern, welche ausgesagt hatten, ihre Kinder 15 Stunden oder mehr primär zu betreuen, hatten signifikant höhere Werte in der Schulvorbereitungsskala $(M W=4.21 ; S D=0.45 ; p<.01)$ als die Elterngruppe mit einer primären Betreuungszeit von 11 bis 15 Std. $(M W=4.04 ; S D$ $=0.55)$. Gleiches gilt für die Skala Motorik/Sport $(M W=4.32 ; S D=0.55)$, wobei sich hier zu beiden anderen Gruppen signifikante Unterschiede ergaben (11-bis-15-Std.-Gruppe: $M W=3.99 ; S D=0.57$; weniger-als-10-Std.-Gruppe: $M W=3.54 ; S D=0.54)$. Auch in der Skala Kunst/Musik zeigt sich fast das gleiche Bild: Die Eltern der Gruppe von 15 Stunden oder mehr betreuten Kinder $(M W=3.74 ; S D=0.56)$ hatten signifikant höhere Erwartungen $(p<.01)$ als die Eltern der beiden anderen Gruppen (11-bis-15-Std.-Gruppe: $M W=3.47 ; S D=0.86$; weniger-als-10-Std.-Gruppe: $M W=3.34 ; S D=0.64$ ). Für die Subskalen soziales Verhalten und Anpassungsfähigkeit ergaben sich in Bezug auf die primäre Betreuungszeit keine signifikanten Unterschiede in den elterlichen Erwartungshaltungen.

\section{Ausbildung der Eltern}

Die Varianzanalysen förderten auch signifikante Effekte der verschiedenen elterlichen Ausbildungsniveaus (obligatorische Schule, Berufslehre, höhere Fachschule, Fachhochschule/Universität/ETH) auf die fünf abhängigen Variablen $(F(12,400)=26.64 ; p<.05)$ zutage. Auch die für jede abhängige Variable durchgeführte Analyse eruierte Effekte sowohl auf die Erwartungshaltungen in Bezug auf die Schulvorbereitung, $(F(12,400)=14.73 ;<.01)$, auf die motorisch/sportlichen $(F(12,400)=25.76 ; p<.01)$ sowie auf die musikalisch-künstlerischen Fähigkeiten $(F(12,400)=25.98 p<.05)$. Diese Befunde wurden wiederum in einem paarweisen Vergleich der Mittelwertsunterschiede getestet. In jedem dieser drei Fälle hatten Eltern mit einem akademischen Abschluss signifikant anspruchsvollere Erwartungen als Eltern aller anderen Ausbildungsgruppen.

Tabelle 5: EAS-Mittelwerte (MW), Standardabweichungen (SD) und Werte der Varianzanalysen für die Ausbildung der Eltern

\begin{tabular}{|c|c|c|c|c|c|c|c|c|c|c|}
\hline \multirow[t]{3}{*}{ Bereich } & \multicolumn{10}{|c|}{ Ausbildung der Eltern } \\
\hline & \multicolumn{2}{|c|}{$\begin{array}{l}\text { Obligato- } \\
\text { rische Schule }\end{array}$} & \multicolumn{2}{|c|}{ Berufslehre } & \multicolumn{2}{|c|}{$\begin{array}{c}\text { höhere } \\
\text { Fachschule }\end{array}$} & \multicolumn{2}{|c|}{$\begin{array}{l}\text { Fachhochschule, } \\
\text { Universität, } \\
\text { ETH } \\
\end{array}$} & \multirow[t]{2}{*}{$\begin{array}{l}F(12, \\
800)\end{array}$} & \multirow[t]{2}{*}{$\mathrm{p}$} \\
\hline & $M W$ & $S D$ & $M W$ & $S D$ & $M W$ & $S D$ & $M W$ & $S D$ & & \\
\hline Schulvorbereitung & 3.82 & 0.88 & 4.04 & 0.55 & 4.21 & 0.45 & 4.33 & 0.61 & 14.73 & $.007^{* *}$ \\
\hline Motorik/Sport & 3.34 & 0.54 & 3.66 & 0.57 & 3.89 & 0.55 & 4.12 & 0.57 & 25.76 & $.012^{* *}$ \\
\hline Kunst/Musik & 3.34 & 0.64 & 3.37 & 0.86 & 3.44 & 0.56 & 3.33 & 0.69 & 25.98 & $.023^{*}$ \\
\hline Soziales Verhalten & 4.99 & 0.44 & 4.98 & 0.33 & 5.05 & 0.40 & 5.31 & 0.77 & 5.13 & .117 \\
\hline Anpassungsfähigkeit & 4.76 & 0.56 & 4.76 & 0.70 & 4.80 & 0.44 & 4.79 & 0.78 & 7.33 & .08 \\
\hline Total $^{\circ}$ & 4.03 & 0.55 & 4.10 & 0.63 & 4.12 & 0.50 & 4.47 & 0.65 & 26.64 & $.017^{*}$ \\
\hline
\end{tabular}

$N=200$ Mütter; $N=200$ Väter; ${ }^{*} p<.05 ;{ }^{* *} p<.01 ;{ }^{* * *} p<.001$; empirischer Wertebereich $=1.00$ bis 6.00 für Schulvorbereitung, Motorik/Sport sowie Kunst/Musik; empirischer Wertebereich $=3.00$ bis 6.00 für Sozialkompetenz und Anpassungsfähigkeit; ${ }^{\circ}$ additive Skala, gemittelter Wert 


\section{Diskussion und pädagogische Konsequenzen}

In diesem Beitrag stand die Fragestellung im Zentrum, welche Erwartungen Eltern an die Bildung und Erziehung ihrer Vorschulkinder haben, ob es Unterschiede zwischen Vätern und Müttern gibt und ob diese Erwartungen vom Geschlecht und Alter des Kindes sowie von der Ausbildung, dem Haushaltseinkommen und dem Ausmass der primären Betreuungszeit der Eltern beeinflusst werden. Diese Fragen wurden anhand des Datenmaterials einer Schweizer Pilotstudie zur Austestung der Educational Attainment Scale EAS von Rescorla et al. (1991) beantwortet. Nachfolgend sollen die Erkenntnisse anhand der beiden Fragestellungen zusammengefasst werden. $\mathrm{Zu}$ berücksichtigen ist dabei, dass es sich um eine eher bildungsnahe Stichprobe handelte und die Befunde deshalb nur für diese Population gelten.

- Im Hinblick auf die erste Fragestellung nach der Ausprägung der Elternerwartungen lässt sich konstatieren, dass diese - auch im Vergleich mit den Ergebnissen von Hyson et al. (1990) oder Rescorla, Hyson und Hirsh-Pasek (1991), deren Durchschnittswerte jeweils unter 4.20 lagen - mit einem Gesamtwert von 4.25 überdurchschnittlich hoch waren und damit die Bedeutung von Colemans Befund (1988), dass Elternerwartungen eine wichtige Rolle spielen, unterstrichen werden kann. Die höchsten Erwartungen hatten die befragten Eltern in Bezug auf das soziale Verhalten, die Anpassungsfähigkeit sowie die Schulvorbereitung. Allerdings verweist die Standardabweichung von 1.14 auf sehr grosse Unterschiede innerhalb der befragten Gruppe. Was Eltern von ihren Kindern im Hinblick auf den Schuleintritt erwarten, kann somit nicht per se mit «hohen Erwartungshaltungen» etikettiert werden. Differenziert man ferner die Erwartungshaltungen nach Müttern und Vätern, so ergeben sich interessante und bedeutsame Unterschiede: Väter zeigten sich in den Bereichen Schulvorbereitung, Motorik/Sport sowie in Kunst/Musik signifikant anspruchsvoller in ihren Erwartungshaltungen als Mütter, nicht jedoch in Bezug auf das soziale Verhalten und die Anpassungsfähigkeit. Obwohl diese Befunde zwar keine Hinweise auf das tatsächliche Erziehungsverhalten zulassen, kann doch angenommen werden, dass Väter bestimmte Bereiche stärker gewichten als Mütter und auch anderes von ihren Kindern fordern. Damit unterstützen diese Befunde tendenziell die Erkenntnisse der Väterforschung, wonach der Vater gerade vor dem Schuleintritt eine zunehmende Bedeutung für das Kind bekommt (Grunow, 2007).

- Die zweite Fragestellung - nach den Determinanten der Elternerwartungen, d.h. nach der Bedeutung von Geschlecht und Alter des Kindes, Ausbildung und Haushaltseinkommen der Eltern sowie primärer Betreuungszeit - lässt sich wie folgt beantworten: Während die befragten Eltern keine geschlechtsspezifischen Erwartungen an ihr Kind hatten, zeigten sich dagegen deutliche Unterschiede in Bezug auf das Alter. Dass die Erwartungen an die sechsjäh- 
rigen Kinder, also kurz vor Schuleintritt, deutlich höher waren als vorher, verweist auf die Bedeutung, die Eltern diesem Übergang beigemessen haben. Allerdings gilt es auch hier die grosse Streuung der Befunde zu berücksichtigen. In besonderem Mass beeinflussten jedoch die primäre Betreuungszeit und das Ausbildungsniveau die Erwartungen, nicht jedoch das Haushaltseinkommen: Eltern, die besonders viel Zeit für die primäre Betreuung ihres Kindes aufwendeten, und solche mit akademischem Ausbildungshintergrund hatten insgesamt die höchsten Erwartungen. Damit unterstreichen auch diese Befunde sowohl die Aussage Colemans (1998), wonach die gemeinsam verbrachte Zeit eine wichtige Determinante ist im Hinblick auf Elternerwartungen, als auch die berichteten Forschungsergebnisse von Craig (2006) oder Walter und Künzler (2002), die eine intensive primäre Betreuung vor allem mit akademischen Elternhäusern in Verbindung bringen.

Mit Blick auf die eingangs erwähnte Thematik, dass heute im Zusammenhang mit hohen Elternerwartungen häufig von einer Treibhausatmosphäre gesprochen wird, lässt sich folgende Antwort formulieren: Zwar liessen sich in der vorliegenden Studie deutliche Ansätze von hohen Erwartungshaltungen - allerdings bei einer tendenziell bildungsnahen Stichprobe - reliabel messen, doch erweist sich ihre Bandbreite, insbesondere der auf die Schule bezogenen, als sehr gross. Dazu kommt, dass die hier vorgelegten Befunde keine Antwort auf die Frage erlauben, inwiefern hohe Elternerwartungen mit förderlichen oder eher überfordernden Entwicklungsbedingungen und -umgebungen einhergehen. Da insgesamt keine Studien zu (hohen) Elternerwartungen und Entwicklungssettings vorliegen, bleibt die empirische Untersuchung von "Vorschulkindern im Treibhaus» ein Forschungsdesiderat.

Vor dem Hintergrund dieser Studie gilt es ferner, einige weiteren Einschränkungen zu beachten: Erstens dürfen die hier vorgelegten Ergebnisse aufgrund des explorativen, deskriptiven und auf bestimmte Variablen eingeschränkten Designs nicht generalisiert werden. Deshalb kann auch nicht beantwortet werden, wie verbreitet die hier berichteten Erwartungsmuster sind und welche Ursächlichkeiten ihnen zugrunde liegen. Zukünftig sind deshalb Forschungen mit grösseren und repräsentativen Stichproben mit möglichst längsschnittlichem Design nötig, um kausale Beziehungsmuster zwischen den Variablen bestimmen und Konklusionen über das Ausmass von Elternerwartungen formulieren zu können. Zweitens steht auch die Frage im Raum, wie ehrlich die Eltern geantwortet haben. So könnte die elterliche Tendenz zur Betonung früher schulvorbereitender und anderer spezifischer Massnahmen durch Mechanismen der sozialen Erwünschtheit beeinflusst worden sein. Da die Daten aus dem Jahr 2008 stammen und zu diesem Zeitpunkt Schulvorbereitung noch als wenig schicklich galt, ist anzunehmen, dass sich die befragten Eltern verstärkt darum bemüht hatten, die Etikette des ehrgeizigen Elternhauses zu vermeiden. Drittens ist die hier verwendete EAS für die Erfassung elterlicher Erwartungshaltungen 
mit Sicherheit nicht erschöpfend genug. Andere, hier nicht erfasste Aspekte von familiären Beziehungen dürften ebenfalls eine Rolle spielen, weshalb anzunehmen ist, dass Elternerwartungen durch eine Anzahl weiterer Faktoren vermittelt sind.

Damit verdeutlicht dieser Beitrag insgesamt, dass in diesem Gebiet noch viel Forschungsarbeit notwendig ist. Wir wissen zu wenig, wie sich elterliche Erwartungshaltungen tatsächlich manifestieren und welches Ausmass sich in welcher Konstellation als förderlich, überfordernd oder unterfordernd erweist. Angesichts des wohl überraschendsten Befundes dieses Beitrags - dass Väter deutlich höhere Erwartungshaltungen an ihr Vorschulkind formulierten als Mütter - wäre die Forschungsarbeit auf diesem Gebiet auch gerade mit dem Ziel voranzutreiben, differenziertere Portraits elterlicher Erwartungsmuster und daraus resultierende Wirkungen auf Schullaufbahn und Schuleintritt von Kindern zu generieren.

\section{Literatur}

Bianchi, S. M. (2000). Maternal employment and time with children: Dramatic change or surprising continuity? Demography, 37, 401-414.

Bourdieu, P. (1982). Die feinen Unterschiede. Kritik der gesellschaftlichen Urteilskraft. Suhrkamp: Frankfurt a. M.

Büchner, A. (2011). Bildung im Kindesalter - eine Privatsache? Oder: Bildung von Anfang an - ein Anspruch ohne Realität? In S. Wittmann, T. Rauschenbach \& H. R. Leu (Hrsg.), Kinder in Deutschland. Eine Bilanz empirischer Studien (S. 74-81). Weinheim: Juventa.

Bundesamt für Statistik. (2009a). Finanzielle Situation der privaten Haushalte in der Schweiz. Neuenburg: Bundesamt für Statistik.

Bundesamt für Statistik. (2009b). Erwerbssituation von Müttern und Vätern. Neuenburg: Bundesamt für Statistik

Coleman, J. S. (1988). Social capital and the creation of human capital. American Journal of Sociology, 94, 95-120.

Craig, L. (2006). Parental education, time in paid work and time with children: An Australian time-diary analysis. British Journal of Sociology, 57, 553-575.

Ditton, H., Krüsken, J. \& Schauenberg, M. (2005). Bildungsungleichheit - der Beitrag von Familie und Schule. Zeitschrift für Erziehungswissenschaft, 8, 285-304.

Dornes, M. (2009). Die Seele des Kindes. Entstehung und Entwicklung. Frankfurt a. M.: Fischer.

Ebbeck, M. (2010). Kulturelle Vielfalt und Bildungserwartungen: Sichtweisen von pädagogischen Fachkräften und Eltern. In W. E. Fthenakis \& P. Oberhuemer (Hrsg.), Frühpädagogik International. Bildungsqualität im Blickpunkt (2. Auflage). Wiesbaden: VS Verlag für Sozialwissenschaften.

Elkind, D. (1988). Das gehetzte Kind. Hamburg: Kabel.

Elkind, D. (1989). Wenn Eltern zuviel fordern - die Rettung der Kindheit vor leistungsorientierter Erziehung. Hamburg: Hoffmann und Campe.

Franiek, S. \& Reichle, B. (2007). Elterliches Erziehungsverhalten und Sozialverhalten im Grundschulalter. Kindheit und Entwicklung, 16, 240-249.

Gallagher, J. M. \& Coche, J. (1987). Hothousing: The clinical and educational concerns over pressuring young children. Early Childhood Research Quarterly, 2, 203-210.

Grossmann, K. \& Grossmann, K. E. (2012). Bindungen - Das Gefüge psychischer Sicherheit (völlig überarbeitete Auflage). Stuttgart: Klett-Cotta.

Grunow, D. (2007). Wandel der Geschlechterrollen und Väterhandeln im Alltag. In H. Rost \& T. Mühling (Hrsg.), Väter im Blickpunkt. Perspektiven der Familienforschung (S. 49- 76). Leverkusen: Barbara Budrich. 
Hills, T. W. (1987). Children in fast lane: Implications for early childhood policy and practice. Early Childhood Research Quarterly, 2, 265-273.

Hirsh, K. Hyson, M. C. \& Rescorla, L. (1990). Academic environments in preschool: Do they pressure or challenge young children? Early Education and Development, 6, 401-422.

Hofferth, S. L. (2001). Women's employment and care of children in the United States. In L. van Dijk. Retrieved November 30, 2012, from http://www.soziologie.uni-wuerzburg. de/na_rep.pdf

Hyson, M., Hirsh-Pasek, K., Rescorla, L., Cone, J. \& Martell-Boinske, L. (1990). Ingredients of parental pressure in early childhood. Journal of applied developmental psychology, $12,347-365$.

Jungbauer-Gans, M. (2004). Einfluss des sozialen und kulturellen Kapitals auf die Lesekompetenz. Ein Vergleich der PISA 2000-Daten aus Deutschland, Frankreich und der Schweiz. Zeitschrift für Soziologie, 33, 375-397.

Klein, O. \& Biedinger, N. (2009). Determinanten elterlicher Aktivitäten mit Vorschulkindern. Der Einfluss von Bildungsaspirationen und kulturellem Kapital. Mannheim: Mannheimer Zentrum für Europäische Sozialforschung.

Künzler, J., Walter, W., Reichart, E. \& Pfister, G. (2001). Gender Division of Labor in Unified Germany. Retrieved November 30, 2012, from http://www.soziologie.uni-wuerzburg.de/ na_rep.pdf

Kurz, K. \& Paulus, W. (2006). Übergänge im Grundschulalter: Die Formation elterlicher Bildungsaspiration. In K.-S. Rehberg (Hrsg.), Die Natur der Gesellschaft. Verhandlungen des 33. Kongresses der Deutschen Gesellschaft für Soziologie in Kassel (S. 5489-5503). Frankfurt a. M.: Campus.

Lamb, M. E. (2004). The father's role: Cross-cultural perspectives. Hillsdale: Erlbaum.

Lareau, A. (2003). Unequal childhoods. Berkeley: University of California Press.

Largo, R. (2009). Babyjahre. Die frühkindliche Entwicklung aus biologischer Sicht. München: Piper, Fachverlag für Sozialwissenschaften.

NICHD-Studie. (2008). Sozialverhalten im Grundschulalter. Kindheit und Entwicklung, 16, 240-249.

Paulus, W. \& Blossfeld, H.-P. (2007). Schichtspezifische Präferenzen oder sozioökonomisches Entscheidungskalkül? Zur Rolle elterlicher Bildungsaspirationen im Entscheidungsprozess beim Übergang von der Grundschule in die Sekundarstufe. Zeitschrift für Pädagogik, 53, 491-508.

Petzold, M. \& Nickel, H. (1989). Grundlagen und Konzept einer entwicklungspsychologischen Familienforschung. Psychologie in Erziehung und Unterricht, 36, 241-257.

Rescorla, L., Hyson, L. C. \& Hirsh-Pasek, C. (1991). Academic instruction in early childhood: Challenge or pressure? San Francisco CA: Jossey-Bass.

Rössel, J. \& Beckert-Zieglschmid, C. (2002). Die Reproduktion kulturellen Kapitals. Zeitschrift für Soziologie, 31, 497-513.

Schilther, M. (2012). Bildungserwartungen und bildungsbezogene Unterstützungsleistungen: Eine längsschnittliche Untersuchung zu Familien mit Migrationshintergrund mit Kindern im Vorschulalter. Unveröffentlichte Masterarbeit. Universität Fribourg: Departement Erziehungswissenschaften.

Schmitt, M. (2009). Innerfamiliale Beziehungen und Bildungserfolg. Zeitschrift für Erziehungswissenschaft, 4, 715-732.

Schmitt, M., Kuger, S., Kluczniok, K \& von Maurice, J. (2010). Familiale Anregung während der frühen Kindergartenzeit. In D. Bühler-Niederberger, J. Mierendorff \& A. Lange (Hrsg.), Kindheit zwischen fürsorglichem Zugriff und gesellschaftlicher Teilhabe (S. 145-166). Wiesbaden: VS Verlag für Sozialwissenschaften.

Schuchart, C. \& Maaz, K. (2007). Bildungsverhalten in institutionellen Kontexten: Schulbesuch und elterliche Bildungsaspiration am Ende der Sekundarstufe. Kölner Zeitschrift für Soziologie und Sozialpsychologie, 59, 640-666. 
Sigel, I. E. \& McGilluddy-DeLisi, A. (1982). Parents as teachers of their children: a distancing behavior model. In A. D. Pellegrini \& T. D. Yawkey (Eds.), The development of oral and written language in social contexts (pp. 71-92). Norwoods, N. J.: Ablex.

Sigel, I. E. (1987). Does hothousing rob children of their childhood? Early Childhood Research Quarterly, 2, 211-225.

Spitzer, M. (2010). Wie lernen Kinder? Gehirn- und Denkentwicklung. In W. Weegmann \& C. Kammerlander (Hrsg.), Die Jüngsten in der Kita (S. 65-80). Stuttgart: Kohlmeyer.

Stamm, M. (2010). Vorschulkinder im Treibhaus? In L. Dunker et al. (Hrsg.), Bildung in der Kindheit (S. 126-131). Seelze: Klett.

Stamm, M. \& Edelmann, D. (2013). Handbuch frühkindliche Bildungsforschung. Wiesbaden: Springer/VS.

van der Werfhorst, H. G. \& Hofstede, S. (2007). Cultural capital or relative risk aversion? Two mechanisms for education inequality compared. The British Journal of Sociology, 58, 391-415.

Walter, W. \& Künzler, J. (2002). Parentales Engagement. Mütter und Väter im Vergleich. In N. F. Schneider \& H. Matthias-Beck (Hrsg.), Elternschaft heute. Gesellschaftliche Rahmenbedingungen und individuelle Gestaltungsaufgaben (S. 95-119). Opladen: Leske + Budrich.

Zick, C. D. \& Bryant, W. K. (1996). A new look at parents' time spent in child care: primary and secondary time use. Social Science Research, 25, 260-280.

Zick, C. D., Bryant, W. K. \& Österbacka, E. (2001). Mothers' employment, parental involvement, and the implications for intermediate child outcomes. Social Science Research, 30, 25-49.

Schlagworte: Elternerwartungen, Vorschulkind, primäre Betreuungszeit

\section{Quelles attentes les parents fondent-ils sur leurs enfants d'âge préscolaire? Une étude empirique sur leur rôle et les facteurs déterminants}

\section{Résumé}

Le comportement des parents exerce une grande influence sur le développement des enfants - c'est un fait maintes fois attesté empiriquement. Cependant, peu d'études portent sur le rôle des attentes que les parents fondent sur leurs enfants, surtout à l'âge préscolaire. Cette contribution présente les résultats issus d'une étude pilote suisse concernant les attentes des parents (200 mères et 200 pères) d'enfants d'âge préscolaire. Selon les résultats, les pères fondent des espoirs beaucoup plus importants que les mères sur le développement d'aptitudes scolaires, motrices, sportives, artistiques et musicales de leurs enfants, tandis qu'ils attachent moins d'importance au développement du comportement social de ces derniers. La variable qui contribue le plus à influencer les réponses parentales est le temps de prise en charge. Les parents passant plus de quinze heures par semaine avec leurs enfants ont des attentes nettement plus importantes quant au développement de leurs aptitudes et comportements que des parents allouant un temps de prise en charge inférieur.

Mots-clés: Attentes parentales, enfant d'âge préscolaire, temps de prise en charge parentale 


\title{
Aspettative dei genitori nei confronti dei bambini in età prescolare. Uno studio empirico sul loro ruolo e sui fattori determinanti
}

\begin{abstract}
Riassunto
E un dato di fatto più volte empiricamente documentato che i genitori, con il proprio comportamento, influenzano enormemente lo sviluppo dei loro figli. Tuttavia esistono ancora pochi studi sul ruolo giocato dalle loro aspettative sui figli in età prescolare. Questo articolo presenta alcuni dati tratti da uno studio pilota svizzero sulle aspettative dei genitori con figli in età prescolare. In un campione di $\mathrm{N}=200$ madri e $\mathrm{N}=200$ padri, questi ultimi mostrano aspettative decisamente più alte nei confronti dello sviluppo di competenze accademiche, motorio-sportive e artistico-musicali dei propri figli, ma non nei confronti dello sviluppo del comportamento sociale. La variabile più importante che influenza le risposte dei genitori è il tempo dedicato all'accudimento primario. I genitori che passano più di quindici ore alla settimana con i propri figli hanno aspettative maggiori nei confronti dello sviluppo comportamentale e delle competenze dei propri bambini rispetto a coloro che vi dedicano meno tempo.
\end{abstract}

Parole chiave: Aspettative dei genitori, bambino in età prescolare, accudimento primario

\section{Parental Expectations of Pre-school Children. An empirical study of their role and their determinants}

\section{Summary}

It is a frequently proven empirical fact that the behaviour of parents has considerable influence on their child development. However, only a few studies examine the role their expectations play in the area of early childhood education. This contribution presents results provided by a Swiss pilot study about the expectations parents (200 fathers and 200 mothers) report to have for their pre-school children. Results show that fathers indicate significantly higher expectations than mothers with regard to the development of the academic, motor, athletic, artistic, and musical skills of their child, but not with regard to his/her social behaviour development. The most important variable influencing parental responses is the time devoted to primary care. Parents spending more than fifteen hours per week with their child, have greater expectations regarding the skills and behaviour development of their child than parents spending less time on primary care.

Keywords: Parental expectations, pre-school child, primary care 